\title{
Thermal Meson properties within Chiral Perturbation Theory
}

\author{
A.Gómez Nicola*, J.R.Peláez ${ }^{\dagger *}$, A.Dobado**‡ and F.J.Llanes-Estrada** \\ * Departamento de Física Teórica II, Universidad Complutense, 28040 Madrid, Spain. \\ †Dip. di Fisica. Universita' degli Studi and INFN, Firenze, Italy. \\ ** Departamento de Física Teórica I, Universidad Complutense, 28040 Madrid, Spain. \\ ${ }^{\ddagger}$ Theory Group, Lawrence Berkeley National Laboratory, Berkeley, CA 94720, USA.
}

\begin{abstract}
We report on our recent work about the description of a meson gas below the chiral phase transition within the framework of Chiral Perturbation Theory. As an alternative to the standard treatment, we present a calculation of the quark condensate which combines the virial expansion and the meson-meson scattering data. We have also calculated the full one-loop elastic pion scattering amplitude at finite temperature and we have unitarized the amplitude using the Inverse Amplitude Method in order to reproduce the temperature effects on the mass and width of the $\rho$ and $\sigma$ resonances. Our results show a clear increase of the thermal $\rho$ width, as expected from previous analysis. The results for the $\sigma$ are consistent with Chiral Symmetry Restoration. We comment on the relevance of our results within the context of Relativistic Heavy Ion Collisions.
\end{abstract}

\section{INTRODUCTION AND MOTIVATION}

The recent development of the Relativistic Heavy Ion Collision (RHIC) program is one of the main motivations to study hadronic matter under extreme conditions of temperature $T$ and density. Here we will consider the meson gas formed when the plasma created after one such RHIC has expanded and hadronized, being in a state where the chiral symmetry is restored. There are strong indications that one could observe the medium effects on such a system. For instance, the dilepton spectrum shows an anomalous behaviour for invariant masses near the $\rho$ mass [1]. The flatness of the spectrum is consistent with a modification of the mass and width of the $\rho$ 's which have time to decay inside the plasma, so that their spectral function acquires thermal corrections due to the interaction with the hot and dense hadron gas [2, 3, 4, 5, 6, 7, 8,9].

In such a system, external momenta and temperature are small compared to the chiral symmetry breaking scale $\Lambda_{\chi} \simeq 1 \mathrm{GeV}$. The relevant degrees of freedom are then the lightest mesons and the interactions among them are best described by a low-energy QCD effective theory based on chiral symmetry. The most general framework comprising the QCD Chiral Symmetry Breaking pattern $S U_{L}\left(N_{f}\right) \times S U_{R}\left(N_{f}\right) \rightarrow S U_{V}\left(N_{f}\right)$ is Chiral Perturbation Theory (ChPT) $[10,11,12,13]$ where any observable can be calculated as an expansion in $p / \Lambda_{\chi}, p$ denoting a meson mass, momenta or the temperature. Thus, ChPT provides model-independent predictions, just by fixing a few low-energy constants (LEC). This program has included the calculation of thermodynamic observables such as the free energy density and the quark condensate, as we will briefly review below. Throughout this paper, we will neglect finite baryon density effects, as ideally in

CP660, Hadron Physics: Effective Theories of Low Energy QCD, edited by A. H. Blin et al. (C) 2003 American Institute of Physics 0-7354-0120-9/03/\$20.00 
the central rapidity region formed after a RHIC.

Since ChPT is intended to provide a systematic low-energy and low- $T$ perturbative expansion, we do not expect it to reproduce resonances. This is strongly related to the fact that ChPT only satisfies unitarity in a perturbative fashion. Over the last few years, there has been a lot of work devoted to enlarge the ChPT applicability range by using unitarization methods, i.e, imposing exact unitarity requirements, like the Inverse Amplitude Method (IAM) [14] or approaches based on Lippmann-Schwinger or BetheSalpeter equations [15]. These methods provide a good agreement with the experimental phase shifts and they are able to generate resonances, like the $\rho$ and the $\sigma$ for the $S U(2)$ chiral symmetry. In addition, they can be extended to include coupled channels, describing successfully all the meson-meson data and resonances in the $S U(3)$ case, up to $1.2 \mathrm{GeV}[16,17]$.

What we will show below is that only requiring chiral symmetry and unitarity one can also describe successfully the thermal behaviour of the $\rho$ and $\sigma$ resonances. For that purpose, one needs first to calculate the thermal pion scattering in ChPT, which has been done in [18]. We shall discuss the main features of such thermal amplitude below. Then, by using the IAM extended to finite $T$, one can construct a nonperturbative thermal unitarized amplitude which, in particular, describes the behaviour of the thermal $\rho$ and $\sigma$ [19]. We will present the results for the thermal mass and width of the $\rho$ and $\sigma$ in that approach, as well as for the $T$-dependence of the effective $g_{\rho \pi \pi}$ vertex. The main implications of our results in the context of RHIC and chiral symmetry restoration will be also discussed below.

\section{THE MESON GAS AND CHIRAL SYMMETRY AT FINITE $T$ WITHIN CHPT}

For the reasons explained above, it is important to provide an accurate description of the low- $T$ meson gas in thermal equilibrium. For instance, the signature of chiral symmetry restoration at $T_{c} \simeq 150-200 \mathrm{MeV}$ should be observed in the thermal evolution of the order parameter, the quark condensate $\langle\bar{q} q\rangle(T)$ from below the transition point. As we have just discussed, ChPT provides a model independent description of the thermodynamic observables, based only on chiral symmetry. The only extra ingredient is the temperature, which is treated as an $\mathscr{O}(p)$ parameter.

The first calculations of the pion gas within ChPT go back to [20], where $\langle\bar{q} q\rangle(T)$ and the thermal dependence of $f_{\pi}(T)$ were calculated up to $\mathscr{O}\left(T^{2}\right)$ (one loop) in the chiral limit. That result already showed a behaviour compatible with chiral symmetry restoration. In [21] a thorough analysis up to $\mathscr{O}\left(T^{6}\right)$ was performed, including the free energy, the quark condensate and an estimate of the thermal effects of free kaons and etas. The $\mathscr{O}\left(T^{4}\right)$ corrections to $f_{\pi}(T)$ have been analyzed in [22] where it has been shown that beyond $\mathscr{O}\left(T^{2}\right)$ one has to consider separately the space and time components of the axial current, so that there are two independent $f_{\pi}^{s, t}$, which, in addition, can be complex. In fact, their imaginary part is proportional to the in-medium pion damping rate while their real parts are related to the deviations of the pion velocity from the speed of light. Other analysis of the thermal pion dispersion law can be found in [23, 24]. 
The analysis of typical nonequilibrium effects such as explosive pion production after a RHIC can be also studied within the ChPT context [25]. It should be pointed out that many of these properties have also been investigated using specific models for lowenergy QCD. The most popular is the $O(4)$ model, which reproduces a critical behaviour already in the mean field approach. Apart from introducing the $\sigma$ explicitly, conventional perturbation theory in the $O(4)$ model breaks down, which has been dealt with at finite $T$ using different nonperturbative approaches [26, 27].

When calculating thermodynamic quantities such as the pressure or the quark condensate from ChPT, the usual approach is to use the Feynman rules of Thermal Field Theory [28] to the order considered. This is particularly cumbersome in the three flavor case if one wishes to include the full dependence on temperature, quark masses and $S U(3)$ interactions. An alternative $[21,29,30]$ is to perform a virial expansion of the pressure as [31]

$$
\beta P=\sum_{i} B_{i}(T) \xi_{i}+\sum_{i}\left(B_{i i}(T) \xi_{i}^{2}+\frac{1}{2} \sum_{j \neq i} B_{i j}(T) \xi_{i} \xi_{j}\right) \ldots
$$

where $i=\pi, K, \eta$. This is a dilute gas expansion in the fugacities $\xi_{i}=\exp \left(-m_{i} / T\right)$. The binary interactions between the different species show up in $[21,29,30]$ :

$$
B_{i j}^{(i n t)}=\frac{\xi_{i}^{-1} \xi_{j}^{-1}}{2 \pi^{3}} \int_{m_{i}+m_{j}}^{\infty} d E E^{2} K_{1}(E / T) \sum_{I, J, S}(2 I+1)(2 J+1) \delta_{I, J, S}^{i j}(E),
$$

where $K_{1}$ is the first modified Bessel function and $\delta_{I, J, S}^{i j}$ are the elastic scattering $i j \rightarrow i j$ phase shifts at $T=0$ (chosen so that $\delta=0$ at threshold) of a state $i j$ with isospin $I$, angular momentum $J$ and strangeness $S$. What makes the virial expansion useful is that the $T$ dependence on thermodynamical observables up to $T \simeq 200-250 \mathrm{MeV}$ can be obtained just from the $T=0$ phase shifts, which have been calculated for all possible meson-meson interactions in SU (3) ChPT to one loop. They can be found for instance in [17]. Note that for the pressure one could even use the experimental phase shifts directly. However, the quark condensate is given by the derivative of the pressure with respect to the quark mass and therefore the analytic dependence of the $\delta^{i j}$ with the different meson masses is needed.

For temperatures much below $150 \mathrm{MeV}$, massive states like kaons and eta are thermally suppressed, typically by the Boltzmann factors $\exp \left(-M_{K} / T\right)$ and, in principle, the suppression is even stronger for the interactions among them and with pions, as (1) shows. For low $T$ it is then reasonable to treat those states as free particles, as it was done in [21]. Moreover, at low $T$ the integrals in (2) are dominated by the phase shifts at threshold. However, for higher temperatures, the effect of massive states becomes increasingly important and, furthermore, the dependence of the interactions with the pion mass can be large so that their contribution to the quark condensate becomes sizable [30]. When the effect of the strange states is taken into account, there are two main questions that can be analyzed: on the one hand, the effect on the non-strange condensate $\langle\bar{u} u\rangle=\langle\bar{d} d\rangle=\langle\bar{q} q\rangle / 2$ (in the isospin limit) of adding another flavor. Since the number of degrees of freedom increases, so it does the entropy and one expects that the 


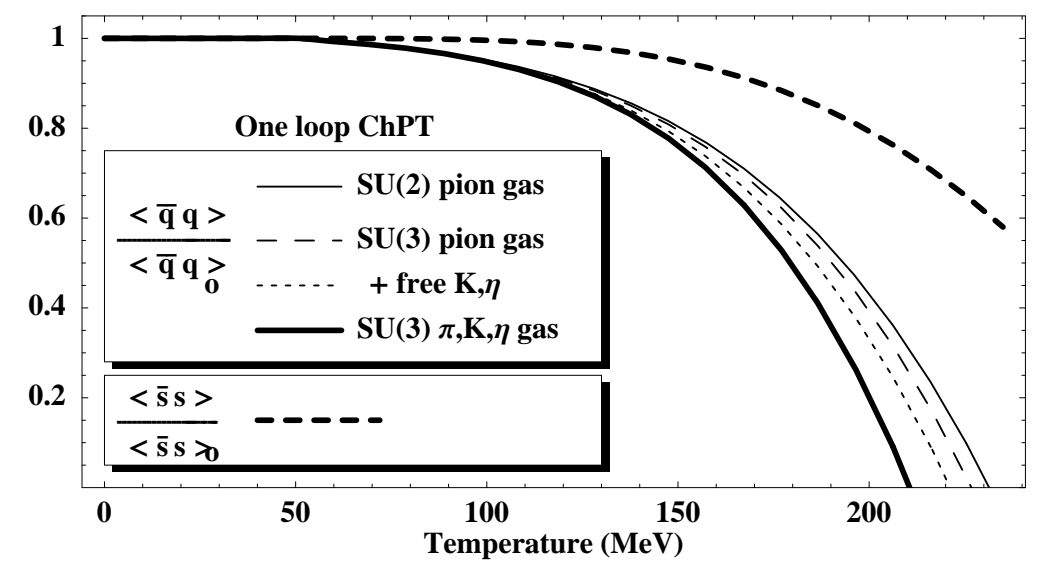

FIGURE 1. The thermal evolution of the $\langle\bar{q} q\rangle$ and $\langle\bar{s} s\rangle$ condensates in ChPT

collective state is closer to "disorder". This would imply a decrease of the critical temperature, as it is indeed observed in lattice calculations [32], although with gluons and massless quarks. On the other hand, one can study the thermal evolution of the strange condensate $\langle\bar{s} s\rangle$. Since $m_{s} \gg m_{u, d}$, one expects that the thermal evolution for $\langle\bar{s} s\rangle(T)$ is slower than for the non-strange condensate. Note that the quark masses play a similar role as external magnetic fields in ferromagnets, so that increasing the external field means that it takes more thermal energy to restore the symmetry. Let us remark that, in contrast to the lattice, the physical masses are easily incorporated in our approach.

We report here on the virial expansion calculation in $S U(3) \mathrm{ChPT}$ that has been done in [30]. The main results for the quark condensates are summarized in Figure 1. First, it should be pointed out that the curves are plotted at most up to the point where the condensates vanish. The ChPT condensates do not vanish above those "critical" values, since they have been obtained from a perturbative series in the temperature. In fact, the results should be trusted only in the region showed in the graphs, as explained above. The thermal evolution of $\langle\bar{q} q\rangle(T)$ is shown in different approximations, namely, considering only pions in $S U(2)$ or $S U(3)$ (their tiny difference comes from the $\mathscr{O}\left(p^{4}\right)$ phase shifts), adding free kaons and etas [21] and, finally, considering the full $S U(3)$ interactions [30]. Note also that these interactions (basically only $\pi K$ and $\pi \eta$ are important at these temperatures) yield a larger effect than naively expected. The reason is that they depend strongly on $m_{\pi}$, which is more sensitive to $m_{u, d}$ than $m_{K}$ or $m_{\eta}$. Taking into account the numerical values of the LEC's with their errors, one gets a reduction of the melting temperature of $T_{m}^{\langle\bar{q} q\rangle S U(2)}-T_{m}^{\langle\bar{q} q\rangle S U(3)}=21_{-7}^{+14} \mathrm{MeV}$, in remarkable agreement with the chiral limit lattice calculations [32], taking into account that we have used the actual meson masses. In addition, we estimate $T_{m}^{\langle\bar{q} q\rangle S U(3)}=211_{-7}^{+19} \mathrm{MeV}$ [30]. The second effect, is also clearly seen in Figure 1: The thermal evolution of the strange condensate from the broken phase is much slower than the non-strange one. From Figure 1, we see that there is still about $80 \%$ left for $\langle\bar{s} s\rangle(T) /\langle\bar{s} s\rangle(0)$ when $\langle\bar{q} q\rangle(T) /\langle\bar{q} q\rangle(0)$ has already melted. Finally, one may wonder about the effect of calculating the integrals in (2) with 
ChPT, whose applicability does not extend to infinity. Indeed, when (2) is evaluated [30] with the phase shifts unitarized with the coupled channel IAM [17], which have a much larger applicability range, the numerical results only change very slightly. Thus, the main conclusions remain the same, since, as we have already commented, the main contribution to the integrals comes from the low-energy region and the IAM agrees with $\mathrm{ChPT}$ at low energy, improving only the high energy behavior.

\section{PION SCATTERING AT $T \neq 0$ IN ONE LOOP CHPT}

If the pion gas is dilute enough, i.e., at very low temperatures, it is reasonable to assume that the only dependence of pion scattering with $T$ can be accounted for through the Bose-Einstein distribution functions and one can ignore the $T$-dependence of the scattering amplitudes. However, that dependence could be important in several contexts. For instance, it has been suggested that an enhancement of pion scattering in the scalar channel near threshold could be an indication of chiral symmetry restoration [27]. In addition, a previous calculation $[9,33]$ of thermal $\pi \pi$ scattering in the Nambu-JonaLasinio model, shows a singular behaviour of the scattering lengths at some critical temperature, which may be related to a Mott transition. Furthermore, if one wishes to extend to finite $T$ the fruitful unitarization program in ChPT to describe resonances, the full calculation of the $\pi \pi$ scattering amplitude to one loop and the extension of perturbative unitarity are essential ingredients.

For the above reasons, it is important to provide a model-independent description of pion scattering for temperatures $T$ well below the chiral scale $\Lambda_{\chi}$. This can be achieved in ChPT. The calculation of the thermal scattering lengths in ChPT to one loop has been done in [34] whereas the calculation of the full thermal amplitude in one loop ChPT has been recently carried out [18]. We will report here the main results and features of that work.

There are two formal aspects regarding the calculation of the scattering amplitude at finite $T$ that are worth pointing out. The first one is that we are considering the thermal amplitude defined by taking $T=0$ asymptotic pion sates, the $T$ dependence coming from the four-point function, calculated using the Feynman rules of Thermal Field Theory in the imaginary-time formalism [28]. Then, one can perform an analytic continuation from discrete frequencies $\omega_{n}=2 \pi n T$ to real energies $E$ as $i \omega_{n} \rightarrow E+i \varepsilon$. Such analytic continuation corresponds to the retarded four-point function in the so called real-time formalism [35]. The retarded functions have a causal and analytic structure [36] suitable to extend perturbative unitarity at $T \neq 0$. The same definition of thermal amplitude has been used in $[9,34]$.

The second point is that the loss of Lorentz covariance inherent to the thermal formalism (due to the choice of the thermal bath rest frame) means that any two-body scattering amplitude with four-momenta $k_{1} k_{2} \rightarrow k_{3} k_{4}$ will depend separately on the variables $\mathbf{S}_{\mathbf{0}}$, $|\overrightarrow{\mathbf{S}}|, \mathbf{T}_{\mathbf{0}},|\overrightarrow{\mathbf{T}}|, \mathbf{U}_{\mathbf{0}}$ and $|\overrightarrow{\mathbf{U}}|$, where $\mathbf{S}=k_{1}+k_{2}, \mathbf{T}=k_{1}-k_{3}$ and $\mathbf{U}=k_{1}-k_{4}$. At $T=0$, the amplitude depends only on the Mandelstam variables $s=\mathbf{S}^{\mathbf{2}}, t=\mathbf{T}^{\mathbf{2}}, u=\mathbf{U}^{2}$ and any $\pi \pi$ scattering amplitude can be related to that of $\pi^{+} \pi^{-} \rightarrow \pi^{0} \pi^{0}$, called $A(s, t, u)$, by isospin and crossing transformations. At $T \neq 0$ and since the temperature does not 


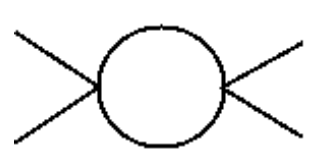

a

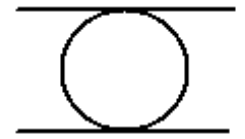

b

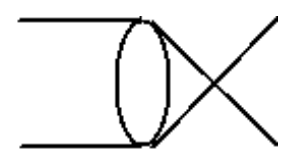

c

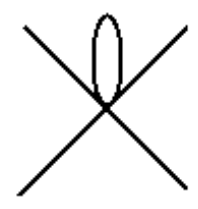

d

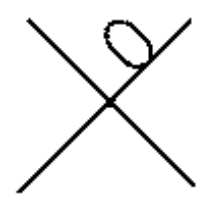

e

FIGURE 2. One loop diagrams contributing to $\pi \pi$ scattering

modify the interaction vertices, the crossing symmetry still holds, but now in terms of $\mathbf{S}, \mathbf{T}$ and $\mathbf{U}$. Therefore, any $\pi \pi$ thermal amplitude can be written in terms of the thermal $A(\mathbf{S}, \mathbf{T}, \mathbf{U} ; \beta)$. The loop diagrams are the same as for $T=0$ and are given in Figure 2 .

The final result for the thermal amplitude can be written as:

$$
A(\mathbf{S}, \mathbf{T}, \mathbf{U} ; \beta)=A_{2}(\mathbf{S}, \mathbf{T}, \mathbf{U})+A_{4}^{p o l}(\mathbf{S}, \mathbf{T}, \mathbf{U})+A_{4}^{\text {tad }}(\mathbf{S}, \mathbf{T}, \mathbf{U} ; \beta)+A_{4}^{\text {uni }}(\mathbf{S}, \mathbf{T}, \mathbf{U} ; \beta),
$$

where $A_{2}$ is tree level contribution coming from the $\mathscr{O}\left(p^{2}\right)$ lagrangian (the nonlinear sigma model) while $A_{4}^{\text {pol }}$ contains both the tree level $\mathscr{O}\left(p^{4}\right)$ contributions plus polynomials coming from the renormalization of the loop integrals. Both $A_{2}$ and $A_{4}^{\text {pol }}$ are temperature independent. The $A_{4}^{u n i}$ term represents those contributions from diagrams $\mathrm{a}, \mathrm{b}, \mathrm{c}$ in Figure 2. They yield the correct analytic structure and will ensure perturbative unitarity in all channels. Finally, the contribution $A_{4}^{\text {tad }}$ accounts for tadpoles like those in diagrams d,e in Figure 2 plus terms coming from diagrams a,b,c proportional to the tadpole integral.

The detailed results for the different contributions above can be found in [18] and we do not give them here for brevity. As a first check of consistency, we recover the $T \rightarrow 0^{+}$ limit of [11]. Furthermore, when the thermal amplitude is projected into partial waves $a_{I J}$ of definite isospin $I$ and angular momentum $J$ (defined in the center of mass frame where the pions are at rest with the thermal bath) we also agree with the results given in [34] for the scattering lengths.

Another important check of consistency, which will be crucial for our analysis in the next section, concerns the imaginary part of the partial waves and perturbative unitarity. At $T=0$, unitarity constraints the partial waves, for $s>4 m_{\pi}^{2}$ and below other inelastic thresholds, to satisfy

$$
\operatorname{Im} a(s)=\sigma(s)|a(s)|^{2},
$$


A

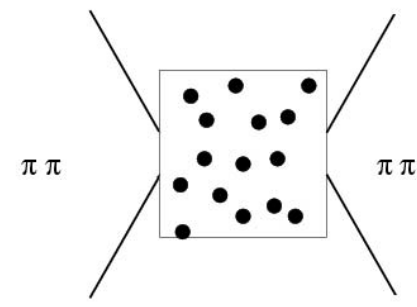

$\left(1+\mathrm{n}_{1}\right)\left(1+\mathrm{n}_{2}\right)$
B

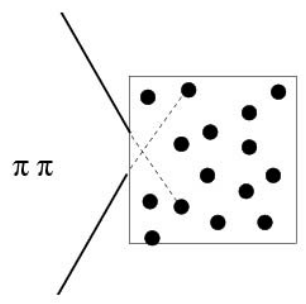

$\mathrm{n}_{1} \mathrm{n}_{2}$

FIGURE 3. Interpretation of thermal bath contributions to the pion scattering phase space. In the process $\mathrm{A}$, the medium enhances the two-pion production, while process $\mathrm{B}$ represents the absorption of the initial pions by the thermal bath.

where $\sigma(s)=\sqrt{1-4 m_{\pi}^{2} / s}$ is the two-pion phase space, whereas the ChPT series only satisfies unitarity perturbatively, i.e.,

$$
\operatorname{Im} a_{2}(s)=0, \quad \operatorname{Im} a_{4}(s)=\sigma(s)\left|a_{2}(s)\right|^{2}, \quad \ldots
$$

It is possible to generalize the relation (5) to the case of any one-loop elastic scattering amplitude at finite $T$. The derivation is given in [18] and follows closely the analysis in [37] of the discontinuity in the self-energy of a particle decaying in the thermal bath. The result for the thermal perturbative unitarity relation is:

$$
\operatorname{Im} a_{2}(s)=0, \quad \operatorname{Im} a_{4}(s ; \beta)=\sigma_{T}\left(\mathbf{S}_{\mathbf{0}}\right)\left|a_{2}(s)\right|^{2}, \quad \mathbf{S}_{\mathbf{0}}>2 m_{\pi},
$$

where

$$
\sigma_{T}(E)=\sigma\left(E^{2}\right)\left[1+\frac{2}{\exp (\beta|E| / 2)-1}\right]
$$

and it has been assumed that only $\pi \pi$ states are available in the thermal bath. Thus, $\sigma_{T}$ is nothing but the thermal phase space, whose origin can be physically interpreted by writing $1+2 n_{B}(E / 2)=\left[1+n_{B}(E / 2)\right]^{2}-n_{B}^{2}(E / 2)$ where $\left.n_{B}(x)=(\exp (x / T)-1)\right)^{-1}$ is the Bose-Einstein distribution function. Written in this way, the first term represents the enhancement of phase space due to the presence of pions in the medium, while the second term accounts for the absorption of the two initial pions by pions in the bath. This is schematically depicted in Figure 3 . We have checked explicitly that the relation (6) holds for our thermal $\pi \pi$ scattering amplitude. Moreover, the partial waves at $T \neq 0$ 

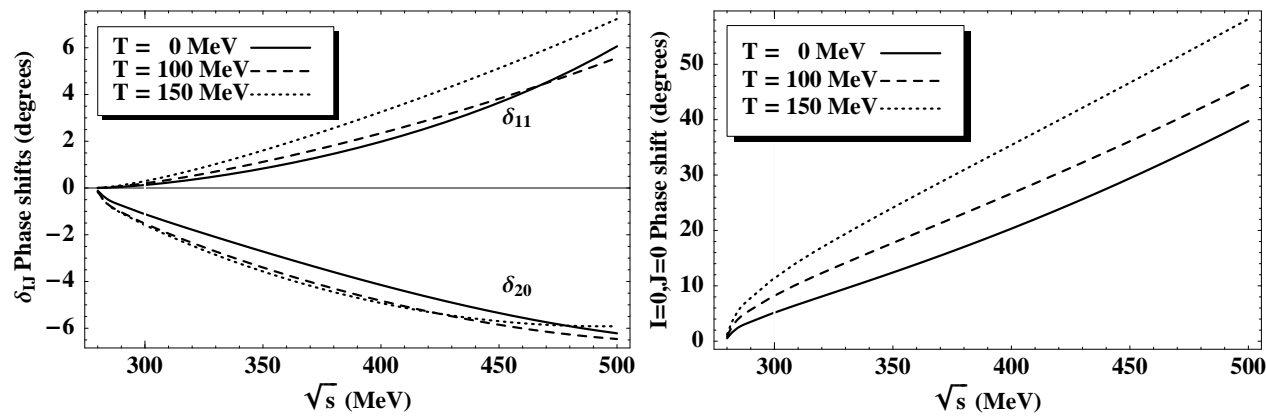

FIGURE 4. Temperature evolution of the phase shifts $\delta_{I J}$ for $I J=11,00,20$.

can be analytically continued to the $s$ complex plane [19] and they display the same analytic structure as the $T=0$ one, i.e, they have a right unitarity cut in the real axis above $s>4 m_{\pi}^{2}$ (the discontinuity across the cut is given by (6)) and a left one for $s<0$. We remark that our results remain valid when the density of states with more than two pions is small. This is equivalent to neglecting higher powers of $n_{B}$ and is therefore similar to the dilute gas approach. For small energies and temperatures, that is, strictly within ChPT, the dilute gas approach is consistent, as we have also seen in the previous section. When the range of energies is extended, as we will do in the next section, one should bear in mind that the range of $T$ where our approach is valid is such that the Bose-Einstein factors still remain small.

Finally, we have plotted the thermal phase shifts in Figure 4. These results deserve some comments. First, we observe that the absolute value of the phase shifts in all channels increases with $T$, while their sign (i.e, the attractive or repulsive nature of each channel) is preserved. Recall that the phase shifts are related to the real part of the amplitude as $\delta_{T} \simeq \sigma_{T}(\sqrt{s})\left[a_{2}(s)+\operatorname{Re} a_{4}(s, \beta)\right]$. The dominant contribution to the phase shifts thermal enhancement is given by the thermal phase space factor $\sigma_{T}$, while the $T$-dependence of the real part of the amplitude is rather weak at low $T$. In particular, we do not observe any significant thermal enhancement for the real part of $a_{00}$, which would be interpreted as chiral symmetry restoration [27]. However, it must be stressed that we are just considering the perturbative amplitude, valid only for low $T$. In the next section, we will consider a nonperturbative extension of the amplitude, which does show a behaviour compatible with chiral symmetry restoration in the 00 channel.

Another important comment regarding our results shown in Figure 4 concerns the 11 channel, i.e, the $\rho$ channel. Recall that, following the hypothesis of resonance saturation, increasing $\delta_{11}$ is equivalent to increasing the ratio $\Gamma_{\rho} f_{\pi}^{4} / M_{\rho}^{5}[11,38]$. Therefore, our results are consistent with a thermal increase of the rho width coming mostly from thermal phase space and an almost constant rho mass, at very low $T$ [3, 4, 8]. This behaviour will be confirmed by our analysis in the next section, where we will find also important corrections at higher temperatures. 


\section{THE THERMAL $\rho$ AND $\sigma$}

The purpose of this section is to show that only from chiral symmetry and unitarity one finds a thermal evolution of the masses and widths of the $\rho$ and $\sigma$ at rest consistent with previous analysis and with the dilepton spectrum observed in RHIC. Unlike the models where the resonances are included as explicit degrees of freedom, we will start from the model-independent pion scattering amplitude in one loop ChPT calculated in the previous section and, imposing exact unitarity, we will construct a nonperturbative amplitude whose poles in the second Riemann sheet in the 00 and 11 channels correspond to the $\sigma$ and $\rho$ respectively.

The exact unitarity relation (4) implies that any partial wave satisfies $a=1 /\left(\operatorname{Re} a^{-1}\right.$ $i \sigma)$ on the real axis below inelastic thresholds. A unitarization method is just one way of approximating $\operatorname{Re} a^{-1}$. The IAM uses the one-loop ChPT and thus ensures that exact unitarity is exactly satisfied and, at the same time, the low-energy predictions of ChPT are preserved. The IAM unitarized amplitude for one channel reads $a^{I A M}=a_{2}^{2} /\left(a_{2}-a_{4}\right)$ [14] and coincides formally with the [1,1] Padé approximant in the $f_{\pi}^{-2}$ expansion.

We have already sketched in the introduction the virtues of the IAM formula at $T=0$ for the description of resonances and the data for higher energies. At $T \neq 0$, we have seen in the previous section that, perturbatively in ChPT and for a dilute gas, the partial waves satisfy thermal unitarity (6)-(7). Therefore, following the same steps as for the $T=0$ case and motivated by the success of the IAM approach, we will consider the unitarized IAM thermal amplitude:

$$
a^{I A M}(s ; T)=\frac{a_{2}^{2}(s)}{a_{2}(s)-a_{4}(s ; T)},
$$

which satisfies the exact elastic unitarity condition

$$
\operatorname{Im} a^{I A M}(s ; T)=\sigma_{T}(s)\left|a^{I A M}(s ; T)\right|^{2} .
$$

The first hint that (8) provides a proper description of thermal resonances comes from the following simple argument. The behaviour of the 11 partial wave in the real axis near $s=M_{T}^{2}$, the $\rho$ mass squared, can be described by a Breit-Wigner parametrization (valid for narrow resonances $\Gamma_{T} \ll M_{T}$ ):

$$
a^{B W}(s ; T)=\frac{R_{T}(s)}{s-M_{T}^{2}+i \Gamma_{T} M_{T}}
$$

where $R_{T}(s)$ can be related to the $\rho \pi \pi$ effective vertex (see below). Now, compare (8) with (10) near $s=M_{T}^{2}$. First, one gets $\operatorname{Re} a_{4}\left(M_{T}^{2}\right)=a_{2}\left(M_{T}^{2}\right)$, which defines the resonance mass and, second, from the unitarity relation (9), we have $\Gamma_{T} M_{T}=-R_{T}\left(M_{T}^{2}\right) \sigma_{T}\left(M_{T}^{2}\right)$. Therefore, if the thermal corrections to $R_{T}$ and to $M_{T}$ were much smaller than those to $\Gamma_{T}\left(R_{T} \simeq R_{0}\right.$ and $\left.M_{T} \simeq M_{0}\right)$ we would simply get

$$
\Gamma_{T} \simeq \Gamma_{0}\left[1+2 n_{B}\left(M_{0} / 2\right)\right],
$$




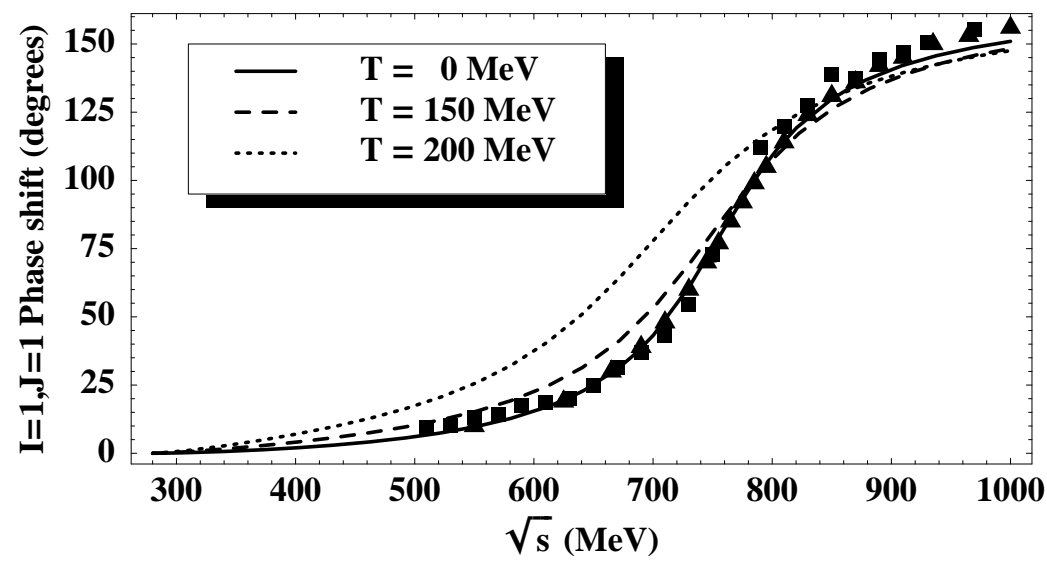

FIGURE 5. $\delta_{11}$ for different temperatures. For the data see [14].

which is the behaviour expected at very low $T$ for a $\rho$ at rest $[4,3,8]$ that we had already anticipated in the previous section. As discussed in [37, 4] and also in the previous section, this result accounts for the difference between the direct decay $\rho \rightarrow \pi \pi$ and the inverse one $\pi \pi \rightarrow \rho$ which is allowed in the thermal bath and is responsible for the dilepton production. Note that in order to derive (11) we have neglected the $T$-dependence in $\operatorname{Re} a_{4}(S ; T)$. Therefore, by using the complete result for the thermal one-loop amplitude discussed in the previous section, we will be able to find, for higher temperatures, the $T$ dependence on $M_{T}, R_{T}$, possible deviations from the low$T$ behaviour (11), and even more importantly, the thermal evolution of both the $\rho$ and $\sigma$ poles in the complex plane, which is the consistent way to generate resonances within ChPT. Recall that a Breit-Wigner description is not valid for the $\sigma$. According to our previous discussion, the upper limit in $T$ to which our approach is valid will be dictated by the condition $n_{B}(M / 2, T)<1$ where $M$ is the mass of the resonance described ( $\rho$ or $\sigma$ ). This gives roughly $T<300 \mathrm{MeV}$ for the $\rho$ and $T<180 \mathrm{MeV}$ for the $\sigma$.

In the above discussion, it is crucial to obtain the analytic continuation of the thermal amplitude to the complex $s$-plane. The details can be found in [19]. The result shows the same right and left cuts structure as the $T=0$ amplitude, the discontinuities across the cuts being $T$-dependent. Once such analytic continuation has been obtained, it can be continued to the second Riemann sheet, using (9), as $a^{I I}(s ; T)=a^{I A M}(s ; T) /[1-$ $\left.2 i \sigma_{T}(s) a^{I A M}(s ; T)\right]$.

Let us first show the results for $\delta_{11}$ for different temperatures, depicted in Figure 5. The $S U$ (2) LEC (see [11] for their definition) we have used are $\bar{l}_{1}=-0.3, \bar{l}_{2}=5.6$, $\bar{l}_{3}=3.4$ and $\bar{l}_{4}=4.3$ and are obtained by fitting the $T=0$ scattering data, which yields $M_{0}=770 \mathrm{MeV}$ and $\Gamma_{0}=159 \mathrm{MeV}$. We see clearly the broadening of the $\rho$ as $T$ increases. This is confirmed by the evolution of the thermal poles, which is shown in Figure 6. The $\rho$ width increases with $T$ while the $\rho$ mass decreases slightly. The $\sigma$ pole deserves some comments. We see that for low $T$ the $\sigma$ width increases and its mass decreases slightly, following a similar behaviour as the $\rho$, i.e, mostly dictated by the 


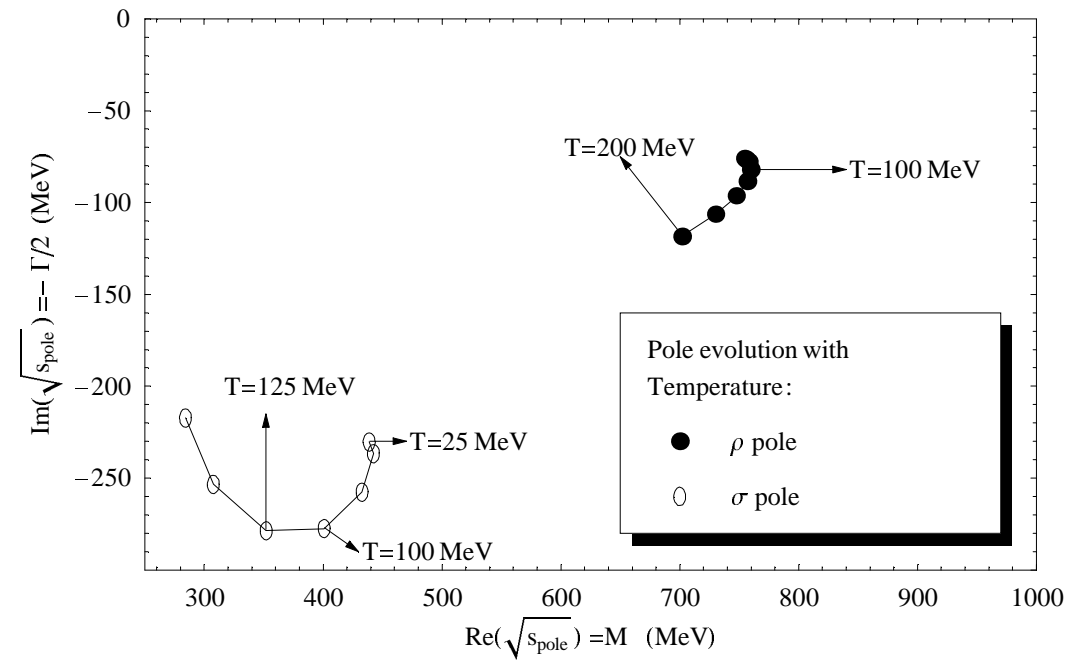

FIGURE 6. Evolution of the $\sigma$ and $\rho$ poles with the temperature.

thermal space factor $\sigma_{T}$. However, for $T>125 \mathrm{MeV}, M_{\sigma}$ decreases more rapidly and $\Gamma_{\sigma}$ starts to decrease. This behaviour has an interesting explanation in terms of chiral symmetry restoration. On the one hand, since the $T$-dependence of $m_{\pi}(T)$ is rather weak up to $T \simeq 200 \mathrm{MeV}$ [24, 27], the decrease of $M_{\sigma}(T)$ points towards $M_{\sigma} \rightarrow m_{\pi}$ and that decreasing is stronger as $T$ increases, unlike the $\rho$ mass. On the other hand, as $T$ increases and $M_{\sigma}$ approaches $2 m_{\pi}$ from above, both the direct $\sigma \rightarrow 2 \pi$ and inverse $2 \pi \rightarrow \sigma$ decays become kinematically disallowed, so that $\Gamma_{\sigma}$ is reduced. At low $T$, the decrease of the mass is much weaker and the phase space contribution dominates, making $\Gamma_{\sigma}$ grow. Similar results and discussion for the $\sigma$ can be found in [27, 39].

Finally, in Figure 7 we have collected our results for the $\rho$. First, we clearly observe a significant deviation (for $T>100 \mathrm{MeV}$ ) between the full result (obtained either from the pole position in Figure 6 or from the phase shift in Figure 5) and the naive thermal phase space prediction (11), stressing the importance of having a full $T$-dependent $\mathrm{ChPT}$ description of $\pi \pi$ scattering. Moreover, although $M_{\rho}$ changes little, consistently with Vector Meson Dominance [2,4], our results show a sizable slight decrease of the $\rho$ mass for $T>150 \mathrm{MeV}$, which seems to be favored by phenomenological analysis of RHIC dilepton data $[6,8]$. In Figure 7 we have also plotted the effective $\rho \pi \pi$ vertex, defined from $R_{T}$ in (10) as $R_{T}=g_{T}^{2}\left(4 m_{\pi}^{2}-M_{T}^{2}\right) / 48 \pi$, from the VMD $\rho \pi \pi$ coupling $[4,7]$ with a thermal $\rho\left(g_{0} \simeq 6.2\right)$. At low temperatures $g_{T} \lesssim g_{0}\left(g_{50} / g_{0} \simeq 0.9991\right)$ in agreement with the chiral analysis in [7]. For higher temperatures $g_{T}$ grows, although the thermal corrections are much smaller than those at finite baryon density [40]. 


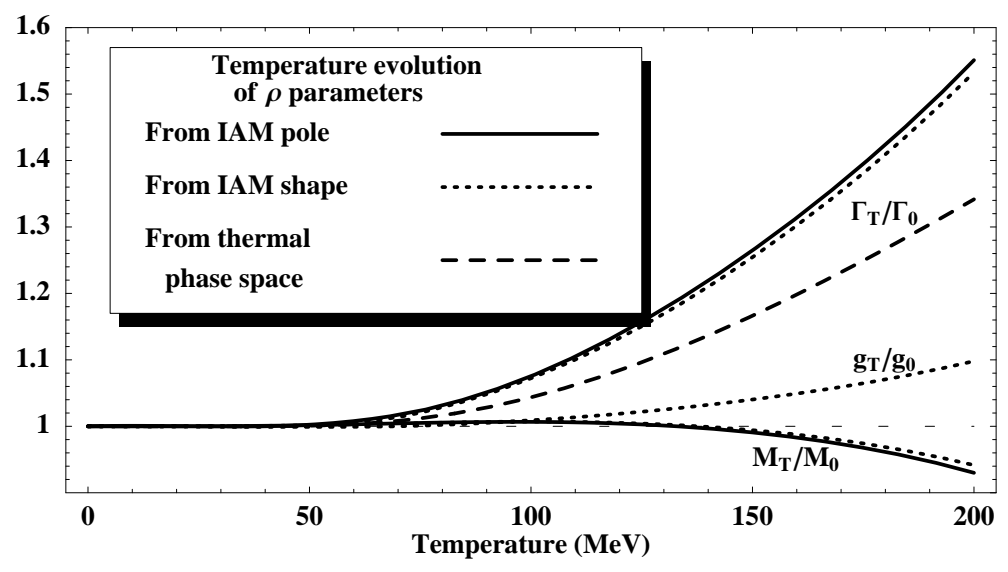

FIGURE 7. Thermal evolution of the $\rho$ mass, width and $\rho \pi \pi$ effective vertex

\section{CONCLUSIONS}

We have reviewed some basic aspects about the ChPT description of the meson gas formed after a Relativistic Heavy ion Collision. In particular, we have shown that one can combine the virial expansion with ChPT in order to obtain the thermal evolution of the quark condensate below the chiral phase transition in the dilute gas regime. That approach allows to use directly all the available information on meson-meson scattering for three light flavors in ChPT. Contrary to lattice studies, in this approach physical masses are easily accounted for. The main effects of considering the strange sector in the condensate are, first, that the strange condensate melts much more slowly than the nonstrange one and, second, that the melting temperature for the non-strange condensate is sizably smaller with three flavors than with two, and that, surprisingly, the $\pi K$ and $\pi \eta$ interactions provide a largest contribution to this effect than free kaons or etas.

We have also shown the results of a recent calculation of the $\pi \pi$ scattering amplitude at finite $T$ in one loop ChPT. The partial waves satisfy an extended version of perturbative unitarity, where the only change with respect to the $T=0$ case is that the phase space is thermally enhanced by the presence of pions in the thermal bath. Thus, the imaginary part of the thermal amplitude has a neat physical interpretation in terms of absorption and induced emission of pion pairs in the thermal bath. The thermal phase shifts are also enhanced with $T$, keeping their attractive or repulsive nature, mainly dominated by the phase space factor.

The thermal pion scattering amplitude can be unitarized using the IAM, following similar steps as for $T=0$. The unitarized partial waves can then be analytically continued to the complex energy plane and their poles in the $I=J=1$ and $I=J=0$ channels correspond to the $\rho$ and the $\sigma$. This approach provides a description of the thermal effects for a $\rho$ and $\sigma$ at rest, only from chiral symmetry and unitarity. We have found that $\Gamma_{\rho}$ increases significantly with $T$. For low $T, M_{\rho}$ and the effective vertex $g_{\rho \pi \pi}$ remain almost constant, which is consistent with neglecting the $T$-dependence in the real part 
of the amplitudes and considering only the contribution from the thermal phase space. However, for higher $T$, the full $T$-dependence in the amplitude has to be taken into account. Thus, for $T>100 \mathrm{MeV}, \Gamma_{\rho}$ acquires significant corrections from the pure thermal space approximation and $g_{\rho \pi \pi}$ increases, while for $T>150 \mathrm{MeV}, M_{\rho}$ shows a sizable decreasing behaviour. As for the $\sigma$ pole, although its interpretation as a resonance is much less clear than the $\rho$, the thermal behaviour of both $M_{\sigma}$ and $\Gamma_{\sigma}$, as obtained from its associated pole, can be understood from chiral symmetry restoration.

Our results agree with several theoretical analysis and are consistent with phenomenological studies of RHIC dilepton data. We have only used chiral symmetry and unitarity as guiding principles, without including the resonances as explicit degrees of freedom. It would be interesting to apply our chiral unitary approach to the production of dileptons from thermal $\pi \pi$ annihilation near the $\rho$ energy, in order to be able to provide more accurate predictions about the observed dilepton spectrum in RHIC. This is just but one of the possible directions that we will pursue in the near future.

\section{ACKNOWLEDGMENTS}

A.G.N and J.R.P wish to thank the organizers of the "2nd International Workshop on Hadron Physics" for their kind invitation. We acknowledge financial support from the Spanish CICYT projects, FPA2000-0956, PB98-0782 and BFM2000-1326. This work was supported in part by the Director, Office of Science, Office of High Energy and Nuclear Physics of the U.S. Department of Energy under Contract DE-AC0376SF00098. J.R.P. acknowledges support from the CICYT-INFN collaboration grant 003P 640.15. A.D. acknowledges support from the Universidad Complutense del Amo Program. Eqns.(4)-(7) and Fig.4 are reprinted from "Finite-temperature pion scattering to one loop in chiral perturbation theory" [18] with permission from Elsevier Science.

\section{REFERENCES}

1. HELIOS-3 collaboration, Nucl. Phys. 590, 127c (1995); CERES (NA45) Collaboration, Nucl. Phys. A661, 23c (1999).

2. Dey, M., Eletsky, V.L. and Ioffe, B.L. Phys. Lett. B252, 620 (1990); Gale, C. and Kapusta, J.I. Nucl. Phys. B357, 65 (1991).

3. Dominguez, C.A., Loewe, M. and Rojas, J.C. Z. Phys. C 59, 63 (1993).

4. Pisarski, R.D. Phys. Rev. D 52, 3773 (1995); Nucl. Phys. A 590, 553c (1995).

5. Haglin, K. Nucl. Phys. A584, 719 (1995).

6. Li, G.Q., Ko, C.M. and Brown, G.E., Phys. Rev. Lett. 75, 4007 (1995); Ko, C.M., Li, G.Q, Brown, G.E. and Sorge, H., Nucl. Phys. A610, 342c (1996).

7. Song, C. and Koch, V. Phys. Rev. C54 3218 (1996).

8. Eletsky, V.L., Belkacem, M., Ellis, P.J. and Kapusta, J.I. Phys. Rev. C64, 035202 (2001).

9. He, Y.B., Hüfner, J., Klevansky, S.P. and Rehberg, P. Nucl. Phys. A630, 719 (1998).

10. Weinberg, S. Physica A96, 327 (1979).

11. Gasser, J. and Leutwyler, H. Ann.Phys.(N.Y.) 158, 142 (1984).

12. Gasser, J. and Leutwyler, H., Nucl. Phys. B250, 465 (1985).

13. Meissner, U.G., Rep.Prog.Phys. 56, 903 (1993); Pich, A., Rep.Prog.Phys. 58, 563 (1995); Dobado, A., Gómez Nicola, A., Maroto, A.L. and Peláez, J.R., Effective Lagrangians for the Standard Model, Springer-Verlag, Berlin, 1997; 
14. Truong, T.N. Phys. Rev. Lett. 61, 2526 (1988); Phys. Rev. Lett. 67, 2260 (1991); Dobado, A., Herrero, M.J. and Truong, T.N., Phys. Lett. B235, 134 (1990); Dobado, A. and Peláez, J.R. Phys. Rev. D47, 4883 (1993); Phys. Rev. D56, 3057 (1997).

15. Oller, J.A. and Oset, E., Nucl. Phys. A620, 438 (1997); Nieves, J. and Ruiz Arriola, E., Phys. Lett. B455, 30 (1999); Nucl. Phys. A679, 57 (2000); Phys. Rev. D63, 076001 (2001).

16. Oller, J.A., Oset, E. and Peláez, J.R., Phys. Rev. Lett. 80, 3452 (1998); Phys. Rev. D59, 074001 (1999); 60, 099906(E) (1999). Guerrero, F. and Oller, J.A., Nucl. Phys. B537, 459 (1999); Nucl. Phys. B602 641 (E).

17. Gómez Nicola, A. and Peláez, J.R., Phys. Rev. D65, 054009 (2002).

18. Gómez Nicola, A., LLanes-Estrada, F.J. and Peláez, J.R., Phys. Lett. B550, $55-64$ (2002).

19. Dobado, A., Gómez Nicola, A., LLanes-Estrada, F.J. and Peláez, J.R. Phys. Rev. C66, 055201 (2002).

20. Gasser, J. and Leutwyler, H., Phys. Lett. B184, 83 (1987).

21. Gerber, P. and Leutwyler, H., Nucl. Phys. B321, 387 (1989).

22. Pisarski, R.D. and Tytgat, M. Phys. Rev. D54, 2989 (1996); Toublan, D. Phys. Rev. D56, 5629 (1997); Martinez Resco, J.M. and Valle Basagoiti, M.A., Phys. Rev. D58, 097901 (1998)

23. Goity, J.L. and Leutwyler, H., Phys. Lett. B228, 517 (1989).

24. Schenk, A., Phys. Rev. D47, 5138 (1993).

25. Gómez Nicola, A., Phys. Rev. D 64, 016011 (2001); Gómez Nicola, A. and Galan-González, V., Phys. Lett. B449, 288 (1999).

26. Pisarski, R.D. and Wilczek, F., Phys. Rev. D29, 338 (1984); Rajagopal, K. and Wilczek, F., Nucl. Phys. B399, 395 (1993); Bochkarev, A. and Kapusta, J.I, Phys. Rev. D54, 4066 (1996); AmelinoCamelia, G., Phys. Lett. B407, 268 (1997); Petropoulos, N., J.Phys.G25, 2225 (1999); Nemoto, Y., Naito, K. and Oka,M., Eur.Phys.J.A9, 245 (2000); Ayala, A. and Sahu, S. Phys. Rev. D 62, 056007 (2000).

27. Chiku, S. and Hatsuda, T., Phys. Rev. D57, R6 (1998), Phys. Rev. D58, 076001 (1998).

28. Le Bellac, M., Thermal Field Theory, Cambridge University Press, London, 1996.

29. Dobado, A. and Peláez, J.R., Phys. Rev. D59, 034004 (1999).

30. Peláez, J.R., Phys. Rev. D66, 096007 (2002).

31. Dashen, R., Ma, S., Bernstein, H.J., Phys. Rev. 187, 345 (1969)

32. Karsch, F., Peikert, A. and Laermann, E., Nucl. Phys. B605, 579 (2001).

33. Quack, E., Zhuang, P., Kalinovsky,Y., Klevansky, S.P. and Hufner,J., Phys. Lett. B348, 1 (1995).

34. Kaiser, N., Phys. Rev. C59, 2945 (1999).

35. Baier, R. and Niégawa, A. Phys. Rev. D49, 4107 (1994).

36. Kobes,R. Phys. Rev. D42, 562 (1990); D43, 1269 (1991).

37. Weldon, H.A. Phys. Rev. D28, 2007 (1983); Ann.Phys. (N.Y.) 214, 152 (1992).

38. Donoghue, J.F., Ramirez, C. and Valencia, G., Phys. Rev. D39, 1947 (1989); Ecker, G., Gasser, J., Pich. A. and de Rafael, E., Nucl. Phys. B321, 311 (1989).

39. Yokokawa, K., Hatsuda, T., Hayashigaki, A. and Kunihiro,T., Phys. Rev. C66, 022201 (2002).

40. Broniowski, W., Florkowski, W. and Hiller, B., Nucl. Phys. A696 870 (2001). 
Copyright $\odot 2003$ EBSCO Publishing 